\title{
Techniques - Ultrasound-guided percutaneous nephrolithotomy: How we do it
}

Darren Beiko, MD, MBA, FRCSC ${ }^{1}$; Hassan Razvi, MD, FRCSC ${ }^{2}$; Naeem Bhojani, MD, FRCSC $^{3}$; Jennifer Bjazevic, MD, FRCSC ${ }^{2}$; David B. Bayne, MD $^{4}$; David T. Tzou, MD ${ }^{5}$; Marshall L. Stoller, $\mathrm{MD}^{4}$; Thomas Chi, $\mathrm{MD}^{4}$

${ }^{1}$ Department of Urology, Queen's University, Kingston, ON, Canada; ${ }^{2}$ Division of Urology, Department of Surgery, Western University, London, ON, Canada; ${ }^{3}$ Division of Urology, Université de Montréal, Montreal, QC, Canada; ${ }^{4}$ Department of Urology, University of California at San Francisco, San Francisco, CA, United States; ${ }^{5}$ Division of Urology, Department of Surgery, University of Arizona College of Medicine, Tucson, AZ, United States

Cite as: Can Urol Assoc J 2019 September 27; Epub ahead of print. http://dx.doi.org/10.5489/cuaj.6076

Published online September 27, 2019

$* * *$

\begin{abstract}
Ultrasonography has emerged as an alternative to fluoroscopy for image-guided percutaneous nephrolithotomy (PCNL) in many countries. Compared to fluoroscopyguided PCNL (F-PCNL), ultrasound-guided PCNL (US-PCNL) is easier to learn and reduces radiation exposure to patients and providers. Despite these advantages, uptake of ultrasound-guided PCNL (US-PCNL) in Canada has been almost nonexistent, largely because it is not incorporated into urologists' training. In this article, we seek to familiarize Canadian urologists with this approach by describing our step-by-step technique for US-PCNL. Additionally, we provide keys to successful implementation of this technique.
\end{abstract}




\section{Introduction}

In Canada, percutaneous nephrolithotomy (PCNL) is performed almost exclusively using fluoroscopy as the image-guiding modality of choice. ${ }^{1,2}$ While a long-established standard for imaging guidance, this technique can be challenging to learn, ${ }^{3}$ limiting the number of urologists who perform PCNL regularly as part of their clinical practice. ${ }^{4}$ To the best of our knowledge, there are no publications originating from a Canadian institution describing ultrasound-guided PCNL (US-PCNL). However, US-PCNL has gained popularity in the United States, ${ }^{5}$ Asia, ${ }^{6}$ Europe, ${ }^{7}$ the Middle East, ${ }^{8}$ South America, ${ }^{9}$ and Africa ${ }^{10}$ over the past several years. Some institutions use ultrasound in combination with fluoroscopy as a strategy to reduce ionizing radiation exposure, ${ }^{11}$ whereas others use it to completely eliminate exposure to radiation during PCNL. ${ }^{12,13}$ Although several variations on access techniques exist among the many urologists performing US-PCNL around the world, ${ }^{14}$ the main overarching goals of making renal access easier for urologists to achieve and reduce ionizing radiation exposure for patients and providers remain the same. When appropriate we offer our patients US-PCNL, and herein we describe our technique and keys to successful implementation of this procedure.

\section{Method}

\section{Patient selection}

When starting any new approach, it is important to carefully select patients to decrease morbidity, optimize patient safety and ensure acceptable outcomes. We therefore recommend selecting generally healthy patients when first adopting this technique. Although obesity is not contraindicated, the extra tissue between the flank and kidney pose a challenge during the steps requiring ultrasonography to optimize imaging. ${ }^{15} \mathrm{We}$ therefore recommend selecting non-obese patients initially and waiting to attempt USPCNL on obese patients until after mastering the technique. Usawachintachit et al showed that successful US-PCNL was associated with the presence of hydronephrosis and the absence of a staghorn calculus. ${ }^{12}$ In summary, the ideal initial candidate for USPCNL is a generally healthy, non-obese patient whose imaging demonstrates a nonstaghorn calculus and at least moderate hydronephrosis. When starting up a new USPCNL program, we recommend seeking out these characteristics in the first several USPCNL cases.

\section{Setup and equipment}

Standard prophylactic intravenous antibiotics are given according to the local antibiogram and per the Canadian Urological Association (CUA) guideline ${ }^{16}$ and/or the American Urological Association (AUA) Best Practices Statement. ${ }^{17}$ Standard general 
anesthesia is administered. An externalized ureteral catheter is placed via a flexible or rigid cystoscope in the supine, cystolithotomy, frog-leg or prone position, depending on surgeon preference and experience. We selectively hang a gravity bag of saline between 40-60 cm above the kidney to allow for passive retrograde filling of the collecting system while draping the patient so that by the time the surgeon is ready for renal access, most collecting systems have artificially-induced mild to moderate hydronephrosis to enable easier renal imaging. Similarly, depending on patient factors, the patient's anatomy and surgeon preference, the PCNL procedure can be performed in either the supine or prone position. Relevant surface anatomy and surgical landmarks used to plan collecting system puncture are shown for the supine and prone positions in Figures 1a and 1b, respectively. In addition to standard equipment required for PCNL, the short list of required additional required capital and disposable equipment and instruments are shown in Table 1.

\section{Procedural steps}

Step 1: Renal ultrasonography

Any portable ultrasound unit can be used with a standard abdominal probe. We prefer to use a convex ultrasound transducer (i.e. a curved-surface probe) at a frequency of 3-5 megahertz (MHz), but a linear ultrasound transducer (i.e. a flat-surfaced probe) may also be used. Although gel is conventionally used for diagnostic ultrasound imaging, to prevent the surgeon's hands from becoming slippery, sterile water or saline periodically applied onto the body wall is sufficient for coupling the probe and obtaining adequate imaging. Initially, longitudinal ultrasound scanning is performed to assess the anatomy of the kidney and perirenal tissues. By convention, the probe is oriented so the upper pole is on the left side of the ultrasound screen. In the longitudinal plane the kidney is often partially obscured by acoustic shadowing from the ribs (Figure 2), so the ultrasound probe is rotated 30-45 degrees to align parallel to the ribs, thus eliminating the acoustic rib shadowing. Posterior calyces are identified and the appropriate entry calyx is selected.

\section{Step 2: Renal puncture}

Percutaneous renal access is achieved using a 20-centimetre, 18-gauge renal access needle. We prefer to use an echogenic needle that facilitates easier visualization of the needle tip, but standard non-echogenic needles may also be visualized and used. One advantage of ultrasound is that, regardless the desired location of puncture, upper, interpolar, and lower pole access can be achieved using a longitudinal view of the needle entering the desired target. ${ }^{14}$ In general, the needle enters the skin approximately $1 \mathrm{~cm}$ away from the caudal end of the probe for a lower pole puncture (Figure 3a) and for an upper pole puncture, the needle typically enters the skin $1 \mathrm{~cm}$ from the cephalad end of the probe, (Figure 3b). In the longitudinal view, the goal is for the needle to be visualized 
in its entirety on the ultrasound monitor as it enters into the skin, crosses through the subcutaneous, fascial, muscular, and perinephric layers through the kidney and into the targeted calyx (Figure 4). While it is sometimes not possible to keep the needle in the same plane and image as it is advanced into the target calyx, gently bouncing the needle provides visual feedback to its location so that minor adjustments can be made. By maintaining the longitudinal view and fanning the ultrasound probe back and forth to identify the location of the needle relative to the targeted calyx, the needle can be redirected into the correct plane relative to the calyx of interest. Once in the collecting system, removal of the needle stylet facilitates visualizing efflux of urine through the needle or aspiration of urine confirms proper positioning of needle tip in the collecting system. Our approach is to generally control the needle freehand without a needle guide in order to preserve maximal flexibility in terms of angle of entry into the kidney. A needle guide can, however, be attached to the ultrasound probe. Use of a needle guide decreases the skill needed to keep the needle in the imaging view, and can therefore facilitate easier renal access, particularly early in the surgeon's learning curve.

\section{Step 3: Guidewire access}

The needle stylet is withdrawn to allow passage of a J-Tip coaxial guidewire through the access needle. We use a J-tip wire to minimize chances of collecting system perforation, but virtually any wire can be used for this step. In order to visualize the wire under ultrasound however, the wire must be a wrapped or lined wire (Figure 5). Hydrophilic wires are often so smooth that they are invisible under ultrasound guidance. The guidewire tip, and its relation to the collecting system, can be localized by gently jiggling the guidewire forward and backward. Using this jiggling motion under ultrasound guidance again provides visual feedback to its location. The guidewire is then secured in place for dilation either within the intrarenal collecting system or advanced down the ureter.

Step 4: Tract dilation

The access needle is withdrawn overtop the guidewire and an appropriate skin incision is made. A 10Fr fascial dilator is then passed over the guidewire. Importantly, the nonechogenic nature of common fascial dilators and safety wire introducers (made of polypropylene or similar synthetic polymer) causes them to obscure visualization of the guidewire as it passes over top the wire (Figure 6). Hence, under ultrasound guidance, it is by ultrasonographically detecting the obscuring of the guidewire that the position of the fascial dilator tip is established. With ultrasound guidance, balloon dilation is much easier to visualize compared to serial dilation, and we recommend utilizing a balloon device for tract dilation and sheath placement. To achieve this, the balloon-dilating catheter is then advanced over the guidewire and its tip is positioned into the collecting 
system. Most balloon tips are slightly echogenic compared to the wire and the goal is to visualize the tip as it advances into the collecting system to verify accurate placement. Jiggling of the guidewire can aid in ensuring proper positioning of the balloon tip, which is ideally within the entry calyx in most cases. Dilation of the balloon is then performed to create the working tract (Figure 7).

Step 5: Sheath placement

Over the balloon dilator, a percutaneous access sheath is carefully advanced under ultrasound-guidance. As this step is difficult to track ultrasonographically, it is recommended the sheath be advanced only until the back end of the balloon is seen through the external aspect of the sheath (Figure 8). This can be measured before placement of the balloon to estimate correct sheath placement. The balloon catheter is then withdrawn, leaving the sheath and guidewire in place.

\section{Step 6: Nephroscopy and stone removal}

Nephroscopy for stone removal proceeds from this point per usual care. This endoscopically driven step is performed using the same technique as standard fluoroscopy-guided PCNL (F-PCNL). With an ultrasound console available in the operating room, ultrasonographic imaging can be used to verify location of the flexible nephroscope and residual stones to ensure visualization and stone clearance of all relevant calyces similarly to how retrograde/antegrade pyelography is used currently.

\section{Step 7: Renal drainage}

We achieve ultrasound-guided renal drainage by placing a 10Fr cope loop pigtail nephrostomy tube and/or 6Fr ureteral stent. These drainage tubes can be visualized as parallel echogenic lines in the collecting system. In cases when tube or stent positioning cannot be confirmed ultrasonographically, cystoscopy and/or nephroscopy may be used as necessary to confirm coiling of ureteral stent in the bladder and coiling of stent/pigtail nephrostomy in renal pelvis, respectively.

\section{Step 8: Nephrostogram}

Contrast-enhanced ultrasound nephrostogram ${ }^{18}$ is performed using a low dose ultrasound contrast agent solution. We inject $1.5 \mathrm{~mL}$ of Optison ${ }^{\mathrm{TM}}$ (GE Healthcare) through the nephrostomy tube, immediately followed by a $5 \mathrm{~mL}$ injection of $0.9 \% \mathrm{NaCl}$ flush, to assess of patency of ureteropelvic junction and ureter.

\section{Discussion}

Since the inception of PCNL in the 1970's, ${ }^{19}$ fluoroscopy has been the most commonly used imaging modality used to guide the surgical steps, as reflected by $86.3 \%$ of PCNL cases being performed with fluoroscopy guidance in the CROES Global PCNL study. ${ }^{20}$ 
Mounting concerns regarding cumulative doses of ionizing radiation imposed on patients with urolithiasis have emerged in recent years. ${ }^{21}$ In an effort to adhere to radiation safety principles such as ALARA (as low as reasonably achievable), intraoperative radiationreducing maneuvers during endourologic cases have been implemented. ${ }^{22-24}$ It follows that perhaps the most obvious advantage of US-PCNL over F-PCNL is the reduction-or in some cases, complete elimination —of ionizing radiation exposure to the patient and members of the operating room (OR) team. Obese patients appear to experience the greatest reduction in ionizing radiation exposure and therefore may benefit most from US-PCNL. ${ }^{25}$ As outlined in Box 1, there are many other potential advantages to using ultrasound to guide PCNL. First, ultrasound can be conveniently performed in any position, ranging from the prone position (the PCNL position most familiar to Canadian urologists) to the supine position. Second, easier identification of a posterior calyx compared to fluoroscopy facilitates a geometrically favorable access to most of the collecting system. In fact, ultrasound often identifies additional posterior calyces that are not readily seen fluoroscopically. Third, ultrasonography provides real-time visualization of renal pathology (i.e. cysts, calyceal diverticula, tumours) and surrounding visceral organs and structures (i.e. liver, spleen, bowels, pleura/lung, great vessels), thereby preventing or decreasing the risk of organ injury. Furthermore, Doppler flow imaging permits identification of intrarenal blood vessels, which in turn can help avoid blood vessel injury and excessive bleeding. Fourth, intraoperative setup is improved because the ultrasound machine requires much less square footage than fluoroscopy machines in the tight-spaced OR working environment. Fifth, when completely fluoroscopy-free USPCNL is performed, physicians and OR personnel may experience less fatigue and back and neck pain/discomfort because there is no need to wear protective lead aprons. Elkoushy et al found that $64.2 \%$ of endourologists have orthopedic complaints, and these complaints correlated with combined annual caseload of ureteroscopy and PCNL procedures. ${ }^{26}$ Sixth, ultrasound facilitates contrast-enhanced ultrasound nephrostogram, a modality that allows assessment of patency of the ureteropelvic junction and ureter postPCNL. ${ }^{18}$ Seventh, of great importance to hospital administrators, US-PCNL has been shown to be associated with lower institutional costs than F-PCNL. ${ }^{27}$ Eighth, residency program directors and fellowship program directors will be reassured by the relatively short learning curve of US-PCNL ${ }^{5,28,29}$. Finally, US-PCNL can be particularly advantageous in certain circumstances such as pediatric cases where radiation exposure is of greater concern, or urinary diversions when retrograde catheterization is not possible.

Despite these numerous advantages, there are some disadvantages to US-PCNL compared to F-PCNL. The most obvious disadvantage is the lack of both familiarity and expertise in the interpretation of ultrasonographic static and dynamic images compared to standard fluoroscopic images for most urologists. At the present time, Canadian 
urologists are much more familiar with performing and interpreting retrograde pyelography and antegrade nephrostography compared to renal ultrasound. Additionally, in 2019 ultrasound still lacks anatomic detail of the collecting system that is provided by standard pyelography, particularly concerning the infundibulo-pelviceal anatomy and angles. This can render certain steps of US-PCNL to be more challenging compared to FPCNL, including guidewire placement, tract dilation, sheath placement and placement of a nephrostomy tube or ureteral stent. However, these disadvantages can be overcome as experience is gained.

Potential disadvantages notwithstanding, ultrasound-guided PCNL has emerged, however, as a reliable, effective and safe option. A systematic review on US-PCNL versus F-PCNL was recently published, and it demonstrated that US-PCNL is as effective as F-PCNL and is associated with lower complication rates. ${ }^{30}$ Certainly the known risks of ionizing radiation have contributed to the trend toward US-PCNL, but as outlined in Box 1 there are several added benefits of using ultrasonography to guide PCNL.

\section{How can I pursue US-PCNL training?}

For urologists performing PCNL who are interested in learning this technique, there are several training options to consider. First, one may pursue a formal or informal observership or preceptorship with a skilled specialist, and although this may sometimes be available locally (for example, with an interested local interventional radiologist), often a visit to an expert at his/her own institution can be beneficial. Alternatively, one may attend a diagnostic and/or therapeutic ultrasonography course to learn the basic skills needed to perform renal ultrasonography in various positions. There has been an increase in the number of PCNL courses that teach ultrasound-guided techniques, created by urologists for urologists and designed to impart this skill set. One practical and inexpensive way of familiarizing oneself with renal ultrasound imaging is to take advantage of any opportunities to perform renal ultrasonography on patients in multiple settings, with their consent (i.e. in clinic, in the emergency room, during shock wave lithotripsy or in the operating room immediately prior to PCNL or other procedures). With this approach, one can overcome the first learning curve in adopting US-PCNL namely mastering the ability to obtain a good image of the kidney and collecting system. Another inexpensive way of training is to use one of the low fidelity simulation models that are available. ${ }^{28}$ Breaking up the mastery of US-PCNL into two skills-diagnostic renal imaging and needle control—is a good framework by which the learner can focus on the two skills separately and thereby move toward learning US-PCNL in a systematic fashion. 


\section{How do I start performing US-PCNL?}

After training, the next step is to implement the technique in one's operating room. In doing so, there are several recommended keys to success. Acquisition of all required capital and disposable equipment and instruments, listed in Table 1, is crucial. Ideally, one can acquire equipment that one is familiar with and/or trained with. Many hospitals and operating room suites have one or more portable ultrasound units, so in general the cost to start up an US-PCNL program should not be prohibitive. ${ }^{27}$ Obtaining support from one's nursing and anesthesiology colleagues should be easy since US-PCNL will decrease everyone's exposure to ionizing radiation and may eventually result in fluoroscopy-less PCNL and elimination of the need to wear lead intraoperatively. One final recommendation applicable when starting up any new clinical program in general is to avoid making any other substantive changes in preoperative or postoperative patient management as the program is being built. By maintaining one's usual care and PCNL clinical pathway, the true effects of US-PCNL on OR team performance and patient outcomes will be discernable.

We recommend a stepwise progression in converting the above 8 steps from fluoroscopy guidance to ultrasound guidance. We recommend progressing by initially using ultrasound to guide step 1 and maintaining fluoroscopy for steps 2 through 8 . Once step 1 has been mastered, then one can progress to step 2, performing steps 1 and 2 using ultrasound guidance and steps 3-8 using fluoroscopy, and so on. Until all 8 steps have been mastered, one will have to employ radiation safety measures and wear radiation protection equipment. This stepwise introduction of ultrasound techniques is expected to result in mastery that will facilitate a safe progression from F-PCNL to completely fluoroscopy-less US-PCNL. Only once one has reliably achieved zero-fluoroscopy PCNL will one become comfortable scrubbing in for PCNLs without wearing radiation safety equipment.

As stated above, proper patient selection is paramount to successful adoption of US-PCNL and implementation of a surgical program. We recommend initial cases involve non-staghorn calculi in healthy, non-obese patients with hydronephrotic kidneys. Hydronephrosis provides a larger volume "target" for needle puncture. Retrograde saline injection can be performed to artificially induce hydronephrosis. Alternatively, contrastenhanced ultrasound nephrostogram can be performed via retrograde ureteral contrast injection to guide puncture when there is no hydronephrosis. ${ }^{31}$

As for any new program, periodic evaluation and re-evaluation is recommended. If technology permits, part of the evaluation may involve video recording, but certainly a review of patient outcomes, particularly compared to one's F-PCNL experience, is recommended. 


\section{Conclusions}

Ultrasound-guided PCNL has been widely shown to be safe and effective. This technique is a "win-win" opportunity; patients as well as OR personnel will be exposed to less ionizing radiation. Although learning US-PCNL may seem like a daunting task initially, the literature has shown that this technique is relatively easy to learn and achievable by any practicing urologist. We recommend it for urologists already performing PCNL who want to increase the safety of PCNL and also for urologists not performing PCNL who are interesting in learning PCNL. 


\section{References}

1. Duvdevani M, Razvi H, Sofer M, et al. Third prize: contemporary percutaneous nephrolithotripsy: 1585 procedures in 1338 consecutive patients. J Endourol 2007;21:824-9.

2. Noureldin YA, Elkoushy MA, Andonian S. Predictors of fluoroscopy time during percutaneous nephrolithotomy: impact of postgraduate urology trainees and S.T.O.N.E. nephrolithometry score. J Endourol 2015;29:542-7.

3. Tanriverdi O, Boylu U, Kendirci M, et al. The learning curve in the training of percutaneous nephrolithotomy. Eur Urol 2007;52:206-11. Epub 2007 Jan 10.

4. Sivalingam S, Cannon ST, Nakada SY. Current practices in percutaneous nephrolithotomy among endourologists._J Endourol 2014;28:524-7. doi: 10.1089/end.2013.0447. Epub 2014 Jan 31.

5. Usawachintachit M, Masic S, Allen IE, et al. Adopting Ultrasound Guidance for Prone Percutaneous Nephrolithotomy: Evaluating the Learning Curve for the Experienced Surgeon. J Endourol 2016;30:856-63.

6. Li J, Xiao B, Hu W, et al. Complication and safety of ultrasound guided percutaneous nephrolithotomy in 8,025 cases in China. Chin Med J (Engl) 2014;127:4184-9.

7. Ramón de Fata F, Pérez D, Resel-Folkersma L, et al. Analysis of the factors affecting blood loss in percutaneous nephrolithotomy: a registry of the Spanish Association of Urology in the supine position. Actas Urol Esp 2013;37:527-32.

8. Inanloo SH, Yahyazadeh SR, Rashidi S, et al. Feasibility and Safety of Ultrasonography Guidance and Flank Position during Percutaneous Nephrolithotomy. J Urol 2018;200:195-201.

9. Batagello CA, Vicentini FC, Marchini GS, et al. Current trends of percutaneous nephrolithotomy in a developing country. Int Braz J Urol 2018;44:304-313.

10. Gamal WM, Hussein M, Aldahshoury M, et al. Solo ultrasonography-guided percutanous nephrolithotomy for single stone pelvis. $J$ Endourol 2011;25:593-6.

11. Zhu W, Li J, Yuan J, et al. A prospective and randomised trial comparing fluoroscopic, total ultrasonographic, and combined guidance for renal access in mini-percutaneous nephrolithotomy._BJU Int 2017;119:612-618. doi: 10.1111/bju.13703. Epub 2016 Nov 28.

12. Usawachintachit M, Tzou DT, Hu W, et al. X-ray-free Ultrasound-guided Percutaneous Nephrolithotomy: How to Select the Right Patient? Urology 2017;100:38-44.

13. Nouralizadeh A, Sharifiaghdas F, Pakmanesh H, et al. Fluoroscopy-free ultrasonography-guided percutaneous nephrolithotomy in pediatric patients: a 
single-center experience. World J Urol 2018;36:667-671.

14. Chu C, Masic S, Usawachintachit M, et al. Ultrasound-Guided Renal Access for Percutaneous Nephrolithotomy: A Description of Three Novel Ultrasound-Guided Needle Techniques. J Endourol 2016;30:153-8.

15. Usawachintachit M, Tzou DT, Washington III SL, Hu W, Li J, Chi T. Ultrasoundguided renal access and tract dilation. Videourology 2016;31:Published online 19 September 2016. https://doi.org/10.1089/vid.2016.0046

16. Mrkobrada M, Ying I, Mokrycke S, et al. CUA Guidelines on antibiotic prophylaxis for urologic procedures. Can Urol Assoc J 2015;9:13-22. doi: 10.5489/cuaj.2382.

17. Wolf JS Jr, Bennett CJ, Dmochowski RR, et al. Best practice policy statement on urologic surgery antimicrobial prophylaxis. Urologic Surgery Antimicrobial Prophylaxis Best Practice Policy Panel. J Urol 2008;179:1379-90. doi: 10.1016/j.juro.2008.01.068. Epub 2008 Feb 20.

18. Chi T, Usawachintachit M, Mongan J, et al. Feasibility of Antegrade Contrastenhanced US Nephrostograms to Evaluate Ureteral Patency. Radiology 2017;283:273-9.

19. Fernström I, Johansson B. Percutaneous pyelolithotomy. A new extraction technique. Scand J Urol Nephrol 1976;10:257-9.

20. Andonian S, Scoffone CM, Louie MK, et al; CROES PCNL Study Group. Does imaging modality used for percutaneous renal access make a difference? A matched case analysis. J Endourol 2013;27:24-8.

21. Elkoushy MA, Andonian S. Lifetime Radiation Exposure in Patients with Recurrent Nephrolithiasis. Curr Urol Rep 2017;18:85.

22. Arenas JL, Baldwin DD. Techniques for Minimizing Radiation Exposure During Evaluation, Surgical Treatment, and Follow-up of Urinary Lithiasis. Curr Urol Rep 2015;16:45.

23. Elkoushy MA, Shahrour W, Andonian S. Pulsed fluoroscopy in ureteroscopy and percutaneous nephrolithotomy. Urology 2012;79:1230-5.

24. Smith DL, Heldt JP, Richards GD, et al. Radiation exposure during continuous and pulsed fluoroscopy. J Endourol 2013;27:384-8.

25. Usawachintachit M, Masic S, Chang HC, Allen IE, Chi T. Ultrasound Guidance to Assist Percutaneous Nephrolithotomy Reduces Radiation Exposure in Obese Patients. Urology 2016;98:32-38. doi: 10.1016/j.urology.2016.04.012. Epub 2016 Apr 22.

26. Elkoushy MA, Andonian S. Prevalence of orthopaedic complaints among endourologists and their compliance with radiation safety measures. J Endourol 2011;10:1609-13. 
27. Hudnall M, Usawachintachit M, Metzler I, et al. Ultrasound Guidance Reduces Percutaneous Nephrolithotomy Cost Compared to Fluoroscopy. Urology 2017;103:52-8.

28. Filippou P, Odisho A, Ramaswamy K, et al. Using an abdominal phantom to teach urology residentes ultrasound-guided percutaneous needle placement. Int Braz J Urol 2016;42:717-26.

29. Song Y, Ma Y, Song Y, et al. Evaluating the Learning Curve for Percutaneous Nephrolithotomy under Total Ultrasound Guidance. PLoS One 2015;13:10:e0132986. doi: 10.1371/journal.pone.0132986. eCollection 2015.

30. Yang YH, Wen YC, Chen KC, et al. Ultrasound-guided versus fluoroscopyguided percutaneous nephrolithotomy: a systematic review and meta-analysis. World J Urol 2018 Sep 22. doi: 10.1007/s00345-018-2443-z. [Epub ahead of print]

31. Usawachintachit M, Tzou DT, Mongan J, et al. Feasibility of Retrograde Ureteral Contrast Injection to Guide Ultrasonographic Percutaneous Renal Access in the Nondilated Collecting System. J Endourol 2017;31:129-134. 
Figures and Tables

Fig. 1. Surface anatomy and surgical landmarks for ultrasound-guided percutaneous nephrolithotomy. (A) Supine; (B) prone.

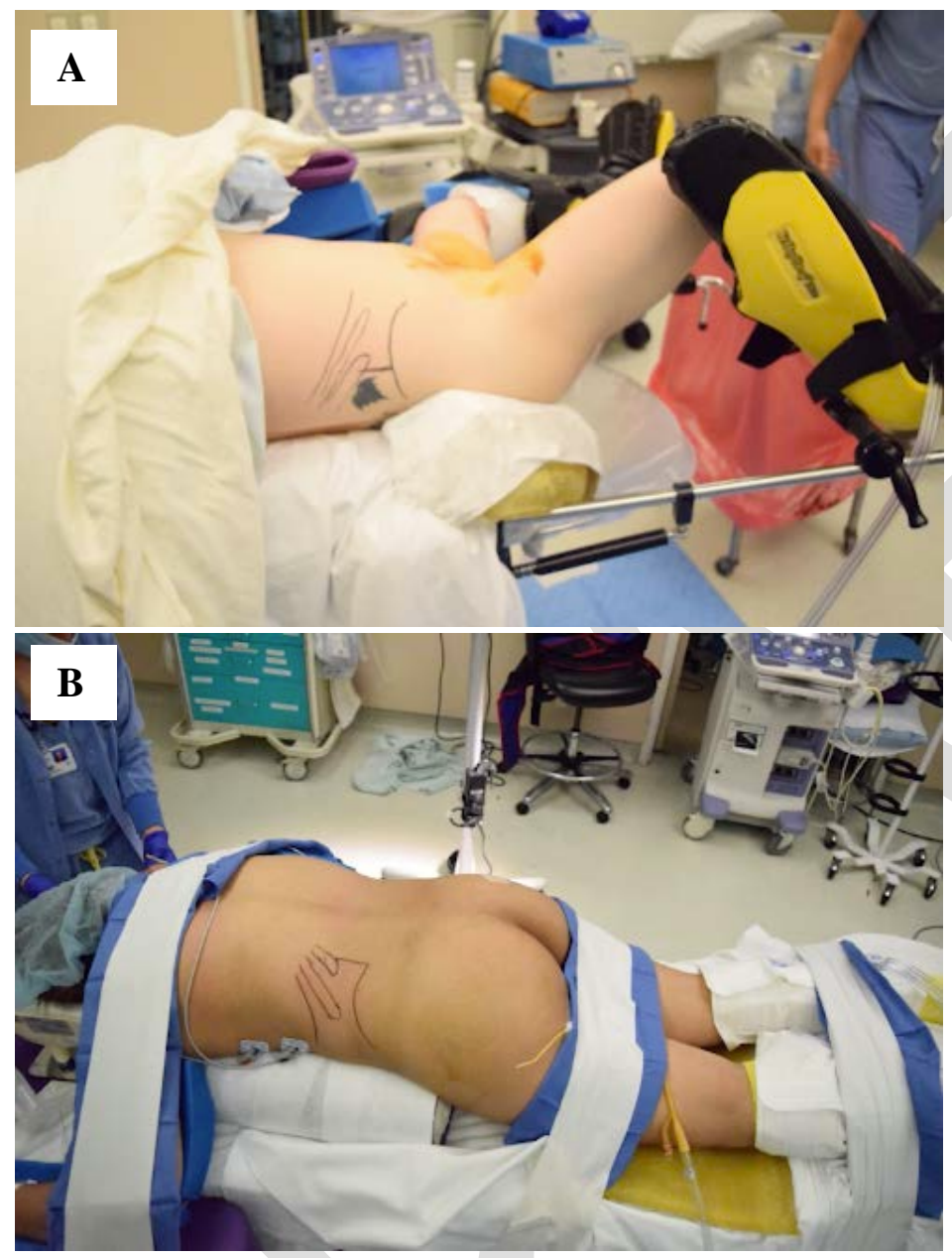


Fig. 2. Acoustic shadows from stones (small arrows) and rib (large arrow) in longitudinal plane.

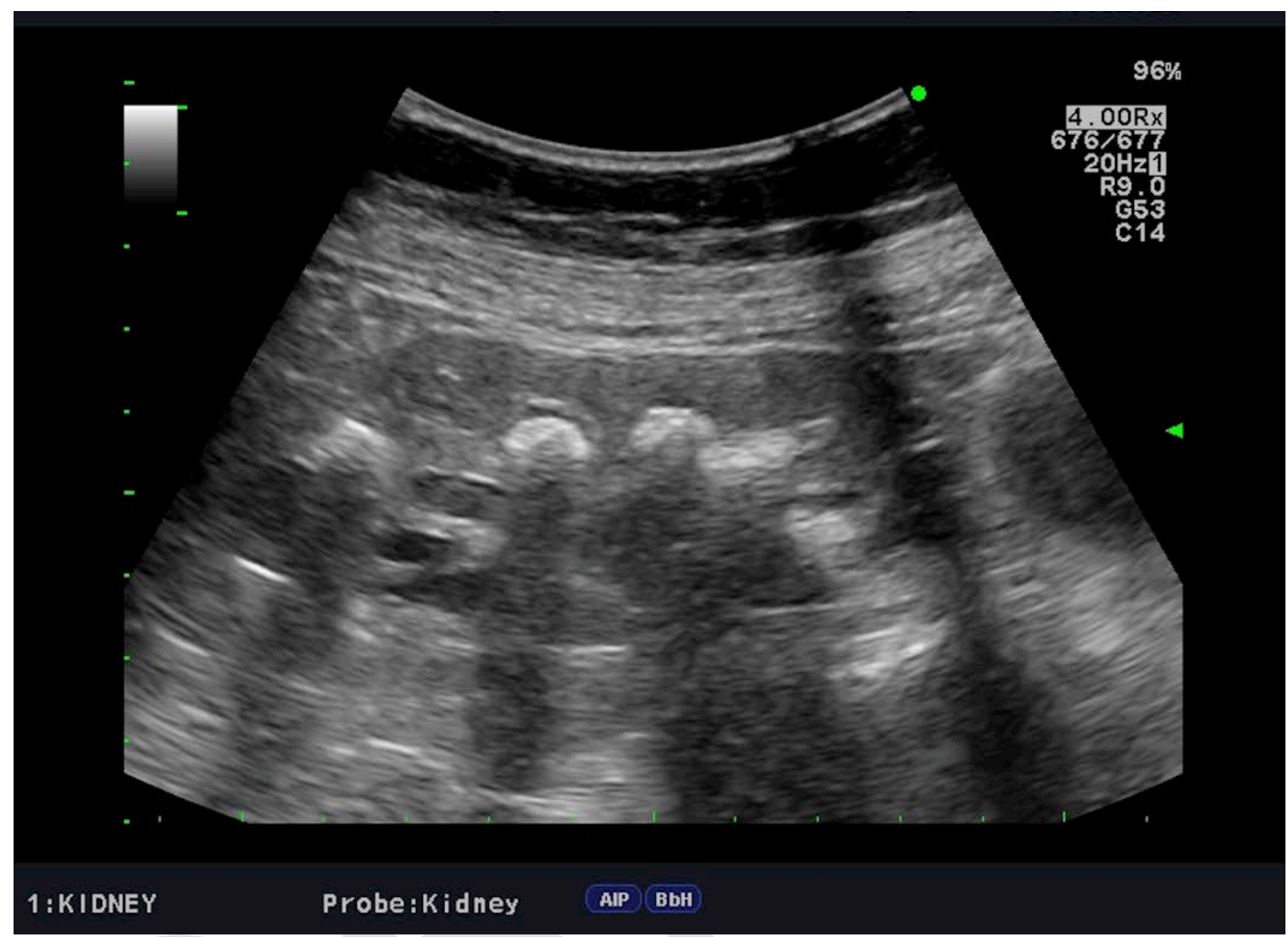


Fig. 3. Needle enters skin $1 \mathrm{~cm}$ from caudal or cephalad end of ultrasound probe for (A) lower and (B) upper pole puncture, respectively.
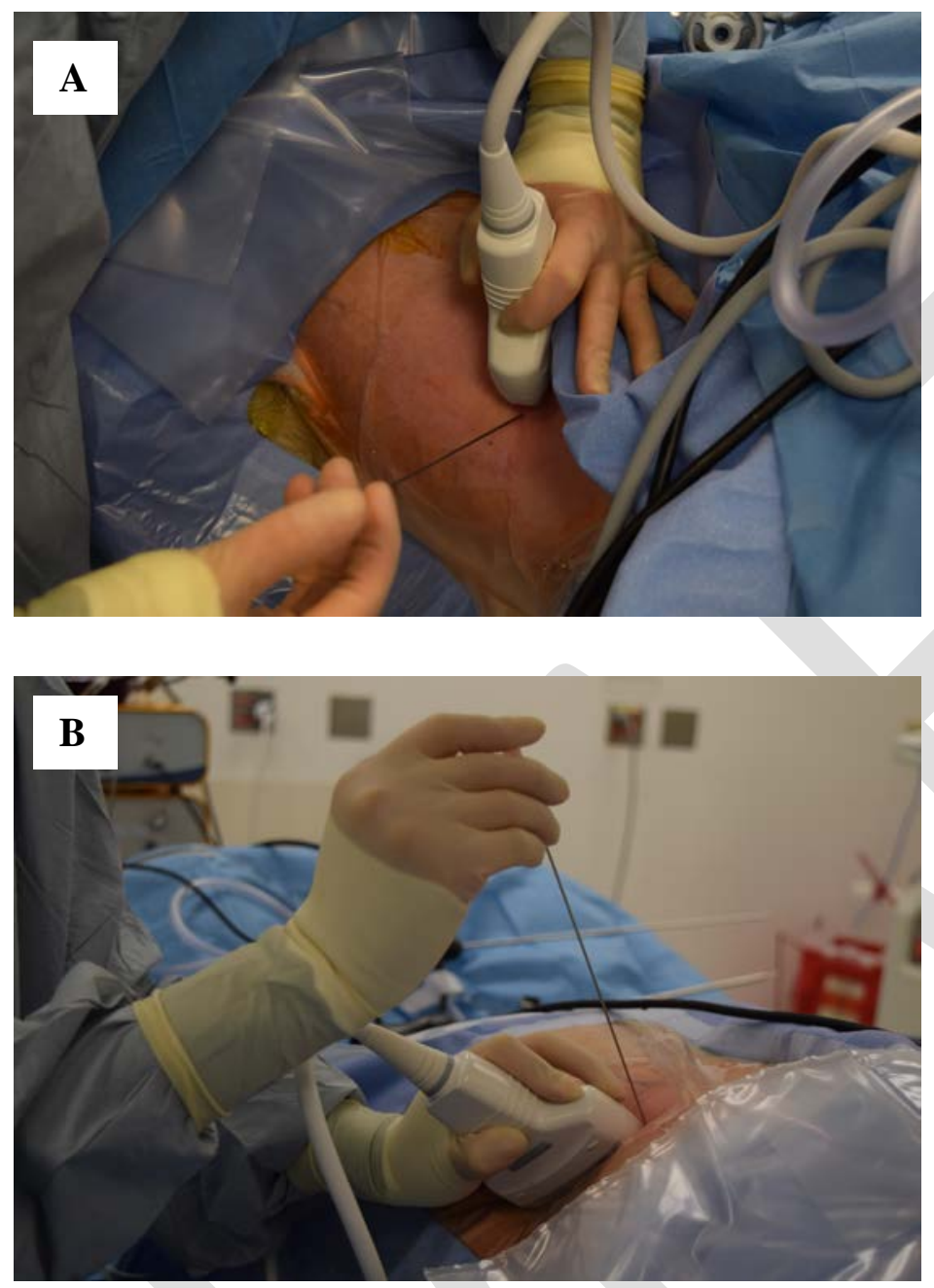
Fig. 4. Visualization of needle (arrow) in its entirely as it traverses all layers into kidney.

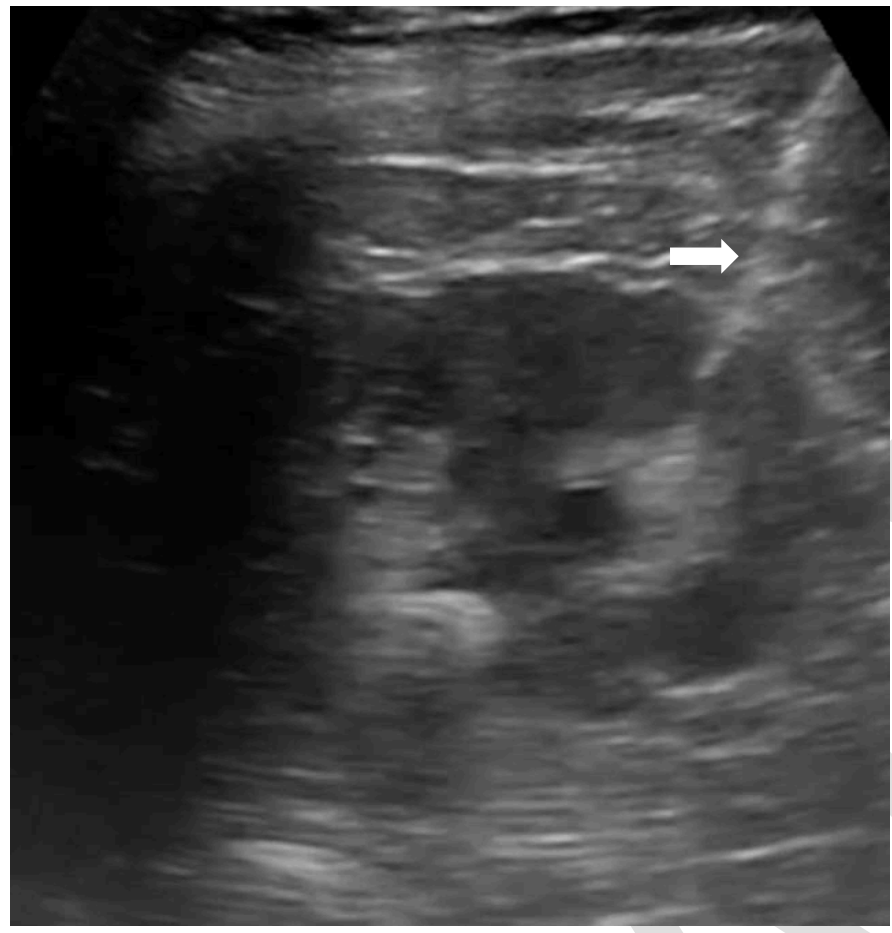

Fig. 5. Non-hydrophilic guidewire readily visualized as it secures lower pole access (same case as Fig. 4).

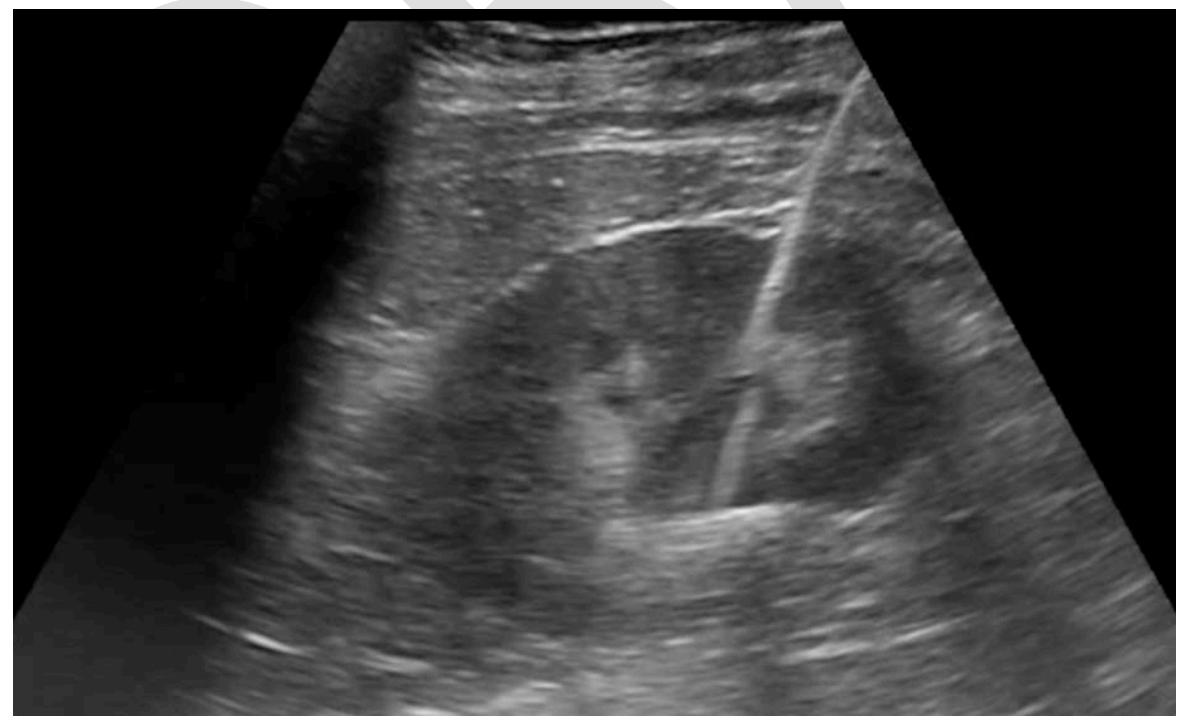


Fig. 6. Position of tip of $10 \mathrm{Fr}$ fascial dilator over guidewire determined by obscuring of guidewire by non-echogenic dilator (same case as Fig. 5, illustrates effect of fascial dilator).

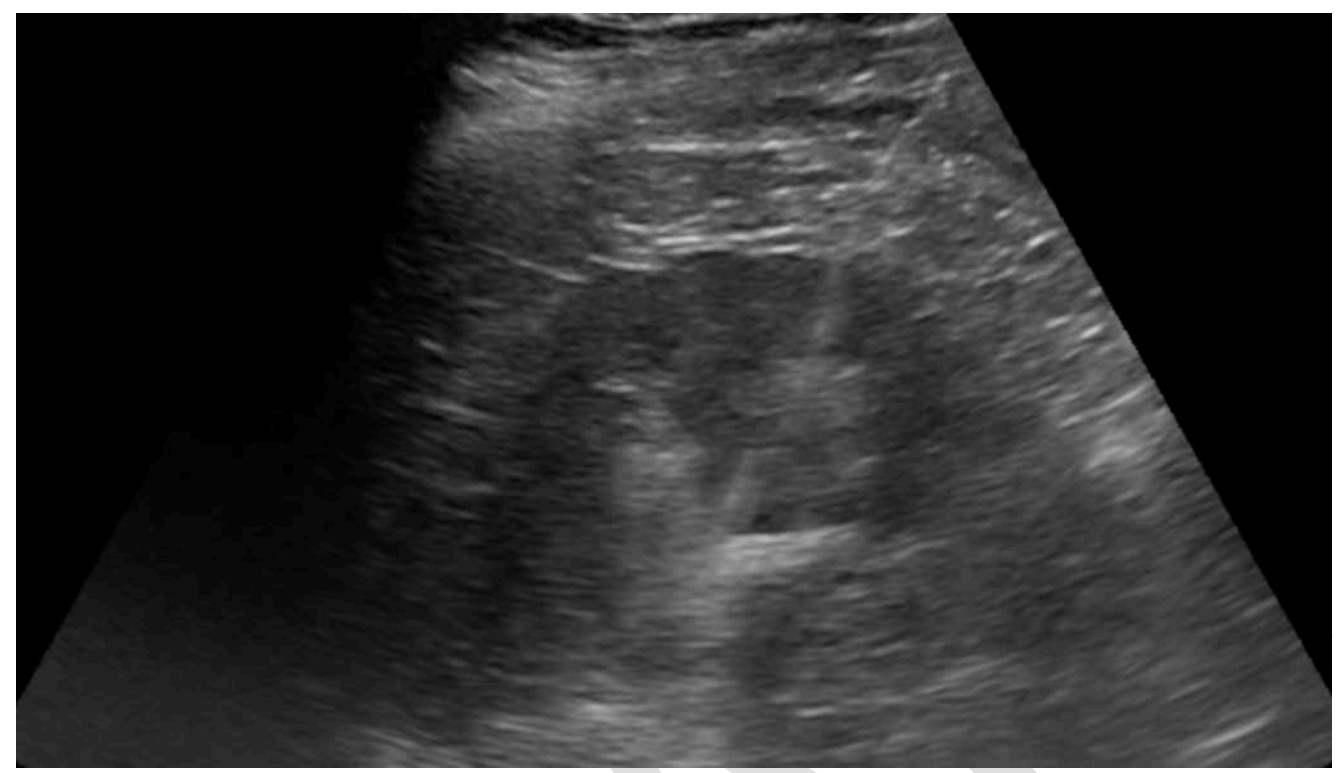

Fig. 7. Balloon dilation of lower pole tract (same case as Fig. 6).

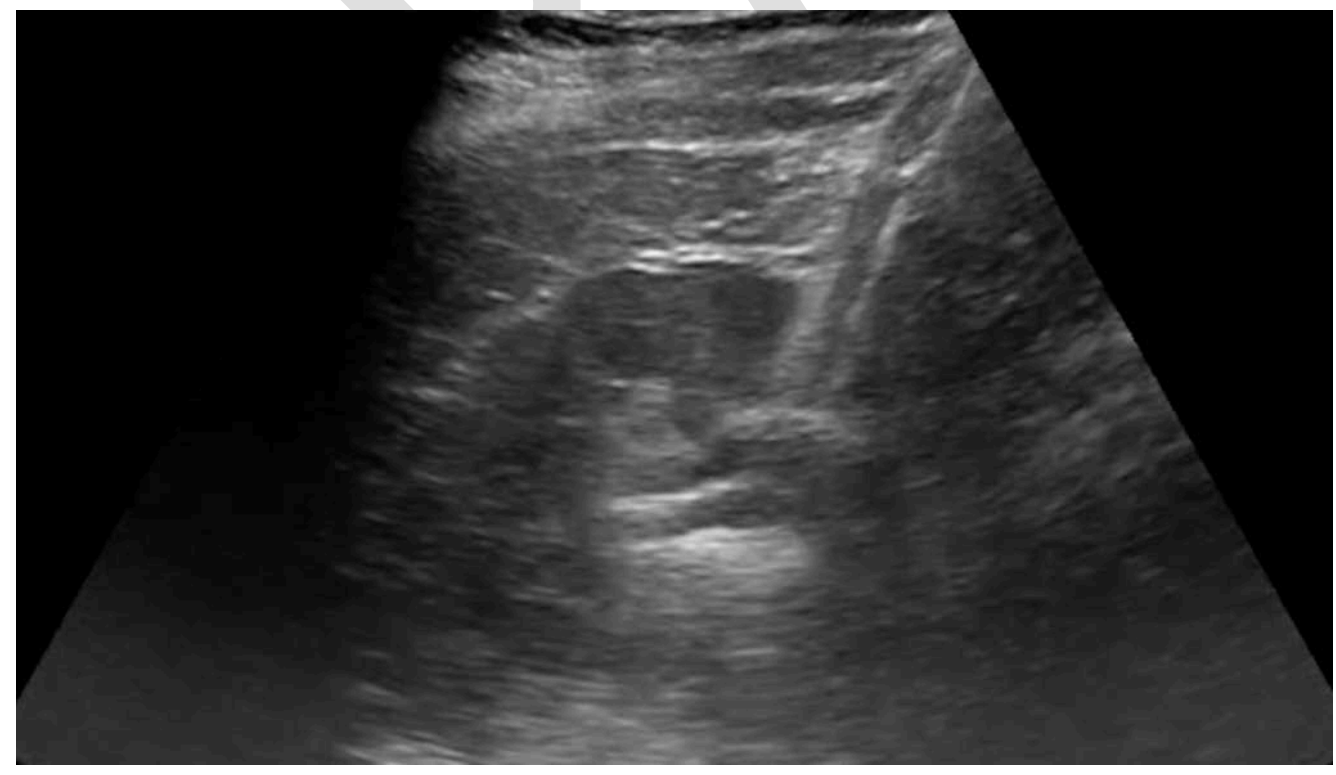


Fig. 8. Percutaneous access sheath advanced until back end of balloon is seen through external aspect of sheath.

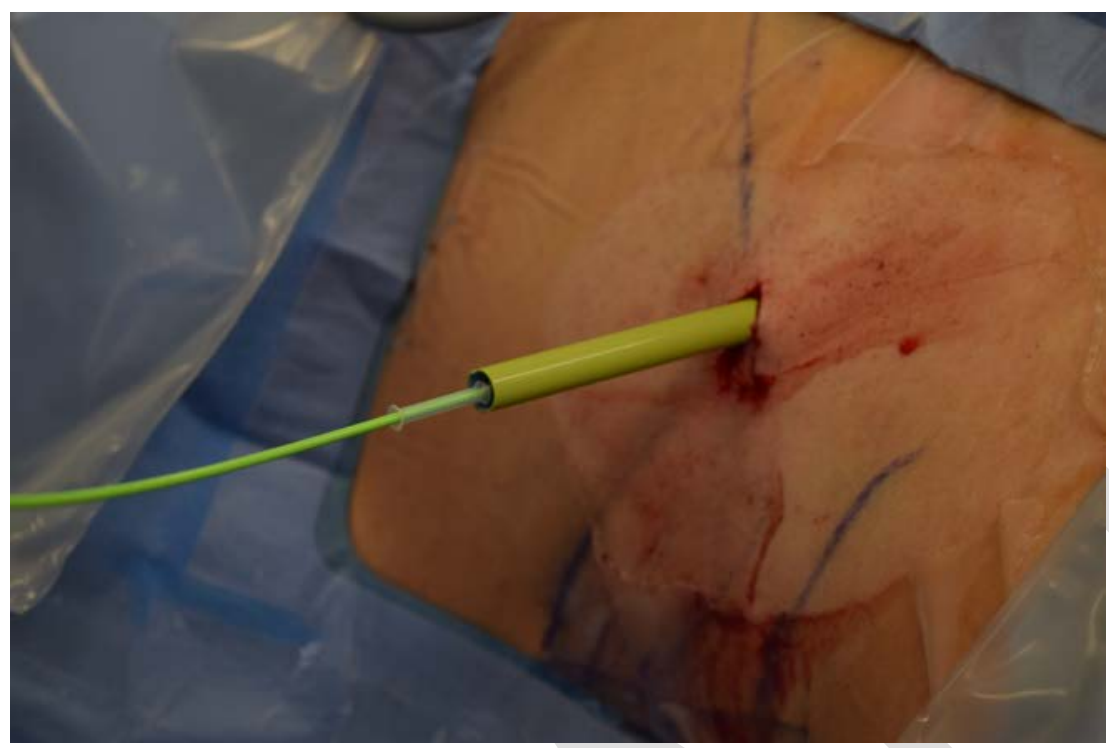

Box 1. Advantages of ultrasound-guided percutaneous nephrolithotomy (US-PCNL)

- Decreased ionizing radiation exposure

- Facilitates supine PCNL

- Easy identification of posterior calyces

- Real-time visualization of renal pathology

- Real-time visualization of surrounding organs/structures

- Doppler flow imaging helps avoid vascular injury

- Takes up less space in OR

- Less physician fatigue by less need to wear lead aprons

- Facilitates contrast-enhanced ultrasound nephrostogram

- Lower cost of capital equipment

- Easier to teach and learn compared to fluoroscopic PCNL 


\begin{tabular}{|l|l|}
\hline \multicolumn{2}{|l|}{$\begin{array}{l}\text { Table 1. Capital and disposable equipment needs for ultrasound-guided } \\
\text { percutaneous nephrolithotomy }\end{array}$} \\
\hline \multirow{4}{*}{ Reusable } & $\begin{array}{l}\text { Portable ultrasound unit } \\
\text { Convex ultrasound transducer } \\
\\
\text { Needle guide }\end{array}$ \\
\hline & Sterile ultrasound console cover \\
& Sterile ultrasound probe cover \\
& Echogenic tip access needle \\
& J-Tip coaxial guidewire \\
& 10Fr fascial dilator \\
& Ultrasound contrast agent solution \\
\hline
\end{tabular}

